Bull. Mater. Sci., Vol. 3, Number 4, December 1981, pp. 435-442. (C) Printed in India.

\title{
On the sintering kinetics of titania doped ceramic lanthanum chromite
}

\author{
K P BANSAL, S KUMARI, B K DAS and G C JAIN \\ Division of Materials, National Physical Laboratory, New Delhi 110 012, India
}

MS received 9 February 1981; revised 9 March 1981

\begin{abstract}
The sintering behaviour of lanthanum chromite with the addition of 1 to 3 $\mathrm{wt}^{\mathrm{O}} \mathrm{\textrm {TiO } _ { 2 }}$ was studied. Densification was examined as a function of sintering temperature and $\mathrm{TiO}_{2}$ concentration. The results showed that pure $\mathrm{LaCrO}_{3}$ could not be densified to $>75 \%$ of the theoretical density, while the densities exceeding $90 \%$ of the theoretical were achieved with $3 \mathrm{wt} \%$ titania addition at $1600^{\circ} \mathrm{C}$.

Metallographic study reveals a normal grain growth following the rate equation $D^{2}-D_{0}^{2}=k t$. Activation energies both for densification and grain growth have been estimated to be $\approx(80 \pm 5) \mathrm{k} \mathrm{cal} / \mathrm{mole}$ in case of $3 \mathrm{wt} \% \mathrm{TiO}$ addition.
\end{abstract}

Keywords. Activation energy; densification; grain growth; lanthanum chromite; sintering kinetics.

\section{Introduction}

Ceramic lanthanum chromite has been emphasized by many workers (Meadowcroft $1969,1972,1973$; Anderson et al 1977, 1978) as the most promising candidate material for MHD systems. Attempts have been made (Leslie Group and Anderson1976; Bansal et al 1980) to prepare dense lanthanum chromites by the addition of certain dopants such as $\mathrm{SrCO}_{3}$ and calcia stabilized Zirconia. Incorporation of magnesia (George and Karkhanawala, 1979) has also been tried to obtain a highly dense and electronically conducting material. St. Jacques et al (1974) have also studied the effect of various additives on the grain growth of $\mathrm{La}(\mathrm{Sr}) \mathrm{CrO}_{3}$.

The primary difficulty encountered in the sintering of $\mathrm{LaCrO}_{3}$ appears to be the volatalization of chromium in oxidizing atmosphere similar to problems identified in the sintering studies of $\mathrm{Cr}_{2} \mathrm{O}_{3}$ and $\mathrm{MgCr}_{2} \mathrm{O}_{4}$ (Halloran et al 1974, Anderson 1974). Callister et al (1979) have recently achieved better sintering of $\mathrm{Cr}_{2} \mathrm{O}_{3}$ with the aid of $\mathrm{TiO}_{2}$. The present study of sintering kinetics and grain growth of $\mathrm{LaCrO}_{3}$ with the addition of $\mathrm{TiO}_{2}$ was also taken up with an aim of improving upon its sinterability.

\section{Experimental}

The material was prepared employing usual ceramic fabrication techniques. Reagent grade $\mathrm{La}_{2} \mathrm{O}_{3}, \mathrm{Cr}_{2} \mathrm{O}_{3}$ and $\mathrm{TiO}_{2}$, in appropriate amounts, were wet mixed by ball milling for $24 \mathrm{hr}$ and the resulting mixture was dried in an oven. The powder was pressed and prefired overnight at $1400^{\circ} \mathrm{C}$ in nitrogen atmosphere. The prefired powder was 
dry ball milled again for $24 \mathrm{hr}$ to get a particle size of $<1 \mu \mathrm{m}$. The powder was then sieved and granulated by means of PVA (Poly Vinyl Alcohol) as binder, and pressed at $8000 \mathrm{psi}$ in the form of discs (14 $\mathrm{mm}$ diameter and $4 \mathrm{~mm}$ thickness).

The tablets so obtained were fired at temperatures ranging from $1500^{\circ} \mathrm{C}$ to $1600^{\circ} \mathrm{C}$ in air for different times. Density measurements were done by water immersion technique (ASTM-71), and for microstructure observations, polished tablets were chemically etched in boiling orthophosphoric acid for a few minutes.

\section{Results}

\section{$3.1 X$-ray diffraction analysis $(X R D)$}

The Debye Scherrer $x$-ray diffractograms of pure and titania doped powder samples of $\mathrm{LaCrO}_{3}$, were obtained. Pure $\mathrm{LaCrO}_{3}$ sample exhibited single phase having an orthorhombic perovskite structure with $a_{0}=5.479 \AA, b_{0}=5.513 \AA, c_{0}=7.756 \AA$, which compares favourably with the published data (Khattak and Cox 1977). The titania doped samples also showed an orthorhombic perovskite lattice structure with a slightly different lattice constants, $a_{0}=5.477 \AA, b_{0}=5.511 \AA, c_{0}=7.752 \AA$.

\subsection{Densification and activation energy for densification}

The influence of $\mathrm{TiO}_{2}$ on the densification behaviour of $\mathrm{LaCrO}_{3}$ is presented in figure 1 , which is a plot of sintered density versus $\mathrm{TiO}_{2}$ concentration at various sintering temperatures; the sintering time being $8 \mathrm{hr}$. At $3 \mathrm{wt} \% \mathrm{TiO}_{2}$ addition, the sintered density sharply increases to $>90 \%$ (of the theoretical) while pure $\mathrm{LaCrO}_{3}$ cannot be densified $>75 \%$ (of the theoretical).

Densification of $\mathrm{LaCrO}_{3}$ versus sintering time and temperature for $3 \mathrm{wt} \% \mathrm{TiO}_{2}$ addition (henceforth known as LCT-3 composition) is presented in figure 2 . The activation energy for densification is calculated from the Arrhenius rate equation

$$
\frac{1}{t}=K_{0} \exp \left(-\frac{Q_{D}}{R T}\right),
$$

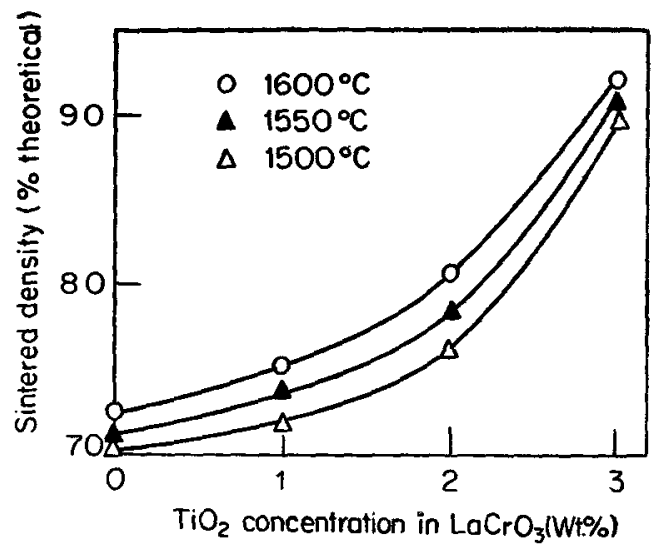

Figure 1. Sintered density as a function of $\mathrm{TiO}_{2}$ concentration for $\mathrm{LaCrO}_{3}$ sintered at various temperatures. 
where $t$ is the sintering time required for the desired densification; $Q_{D}$ is the activation energy for densification which is computed to be $\approx(80 \pm 5) \mathrm{kcal} / \mathrm{mole}$ (figure 3).

\subsection{Grain growth kinetics}

Normal pore free grain growth is obtained for LCT-3 composition and presented in the photomicrographs (figure 4). Figure 5 shows a plot of grain size versus sintering time at various sintering temperatures. The variation of square of grain diameter with sintering time (figure 6) resulted in a straight line, and as such the grain growth follows a rate-equation:

$$
D^{2}-D_{0}^{2}=k t,
$$

where $D_{0}$ is the initial particle size and $K$ is the rate constant.

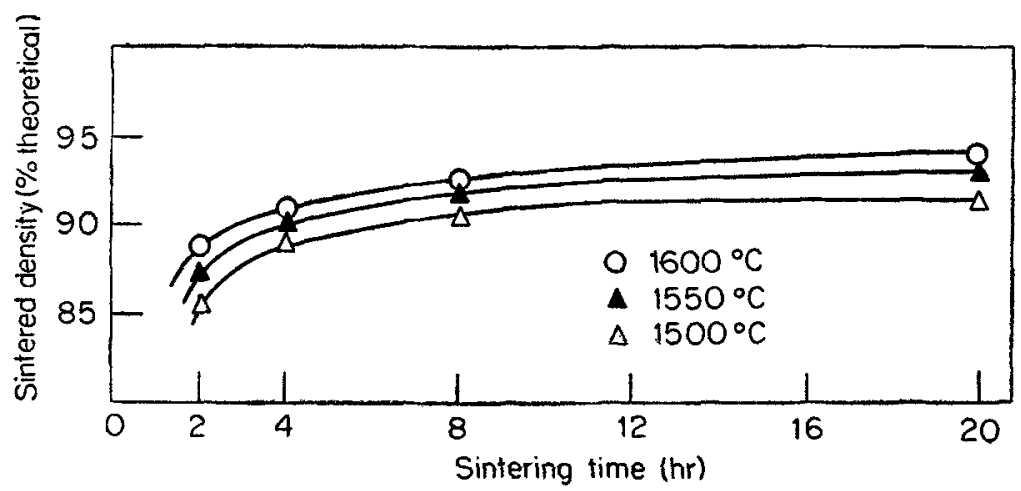

Figure 2. Sintered density versus sintering time plot for LCT-3 composition.

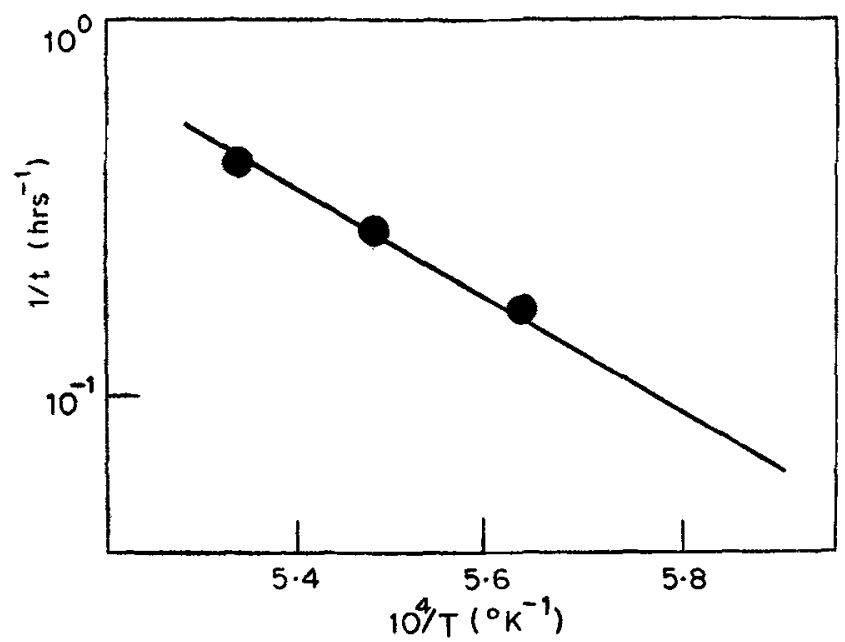

Figure 3. Arrhenius plot of densification rate constant for LCT-3 composition. 
The activation energy for grain growth is estimated following the rate equation

$$
K=K_{0} \exp \left(-\frac{Q_{G}}{R T}\right)
$$

where $Q_{G}$ is the activation energy for grain growth, and it comes out to be $\approx(80 \pm 5)$ $\mathrm{kcal} / \mathrm{mole}$ (figure 7).

\section{Discussion}

The rate of grain growth is decided by the rate at which a curved grain boundary approaches its centre of curvature, which is controlled by the bulk diffusivity of the slowest moving ion in ceramics. The densification rate is on the other hand controlled by the rate of pore removal. Various mechanisms like bulk diffusion, surface diffusion and evaporation-condensation, etc have been proposed for pore removal during sintering. The same values of activation energies for densification and grain growth in the present case indicates that the same mechanism, i.e. the bulk diffusion of the slowest moving ion, controls the rate of densification and grain growth (Stuijts, 1968). The diffusion of cations found at interstitial sites is known to be faster than that of oxygen ions. The activation energy of grain growth and densification can thus be considered as the activation energy for self diffusion of oxygen ions in the perovskite lattice.

An additive may enhance the sintering of a material such as $\mathrm{LaCrO}_{3}$, by stabilizing the oxidation state of the host cation so that the volatile species are not formed, and by modifying the defect structure of one or both host sublattices to accelerate diffusion of the component ions. It is well known (Anderson, 1977, 1978) that the evaporation of $\mathrm{Cr}$ from $\mathrm{La} \mathrm{CrO}_{3}$ by oxidation of $\mathrm{Cr}^{3+}$ to $\mathrm{Cr}^{4+}$ leads to poor densification. Any additive that can stabilize $\mathrm{Cr}$ as $\mathrm{Cr}^{3+}$ would give better densification. Addition of $\mathrm{TiO}_{2}$ leads to such stabilization by the following reactions (George and Karkhanawala, 1979):

$$
\begin{aligned}
& 2 \mathrm{Cr}^{3+}+\frac{1}{2} \mathrm{O}_{2} \rightleftharpoons 2 \mathrm{Cr}^{4+}+\mathrm{O}_{i}^{2-} \\
& 2 \mathrm{Ti}^{4+}+\mathrm{O}_{i}^{2-} \rightleftharpoons 2 \mathrm{Ti}^{3+}+\frac{1}{2} \mathrm{O}_{2} \\
& \mathrm{Ti}^{3+}+\mathrm{Cr}^{4+} \rightleftharpoons \mathrm{Ti}^{4+}+\mathrm{Cr}^{3+}
\end{aligned}
$$

Since the ionic radius of $\mathrm{Cr}^{3+}$ ion $(0.69 \AA)$ is almost equal to that of $\mathrm{Ti}^{4+}$ ion $(0.68 \AA), \mathrm{Ti}^{4+}$ can be easily accommodated substitutionally on the $\mathrm{Cr}^{3+}$ sublattice. However, such a substitution will lead to the creation of cation vacancies on the Cr-sublattice in accordance with valency-charge balances. Presence of cation vacancies will in turn give rise to faster pore mobility if there is a separate path for oxygen diffusion, i.e. through the gaseous phase. A possible mechanism of grain growth by increased pore mobility in the presence of the excess cation vacancies has been suggested by Yan and Johnson (1978), and has explained the grain growth promotion in $\mathrm{TiO}_{2}$ doped $\mathrm{Mn}-\mathrm{Zn}$ ferrites, for the increased pore mobility results 


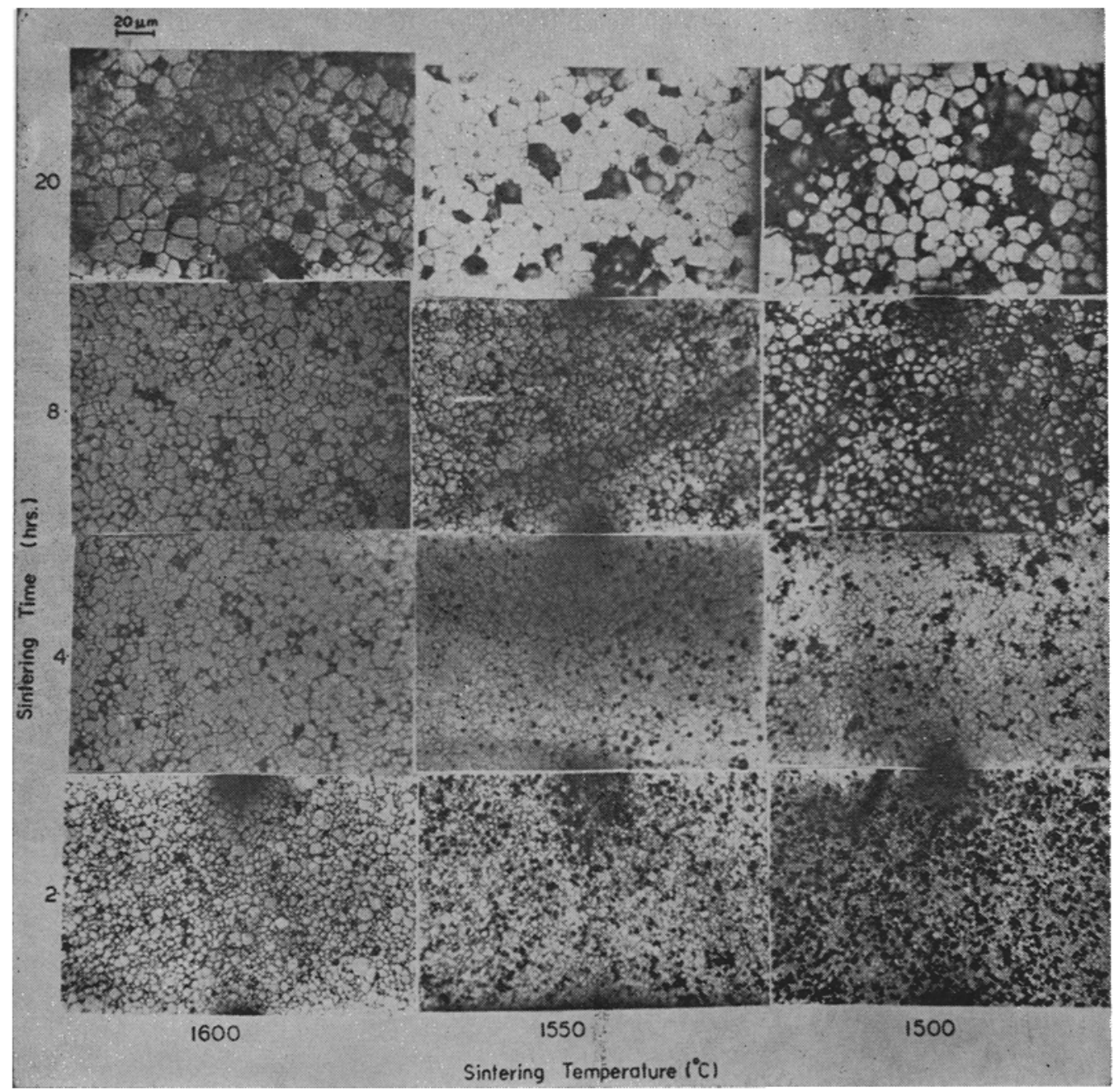

Figure 4. Microstructure of lanthanum chromite doped with $3 \mathrm{wt} \% \mathrm{TiO}_{2}(\mathrm{LCT}-3$ composition) sintered at various temperatures for different times. 


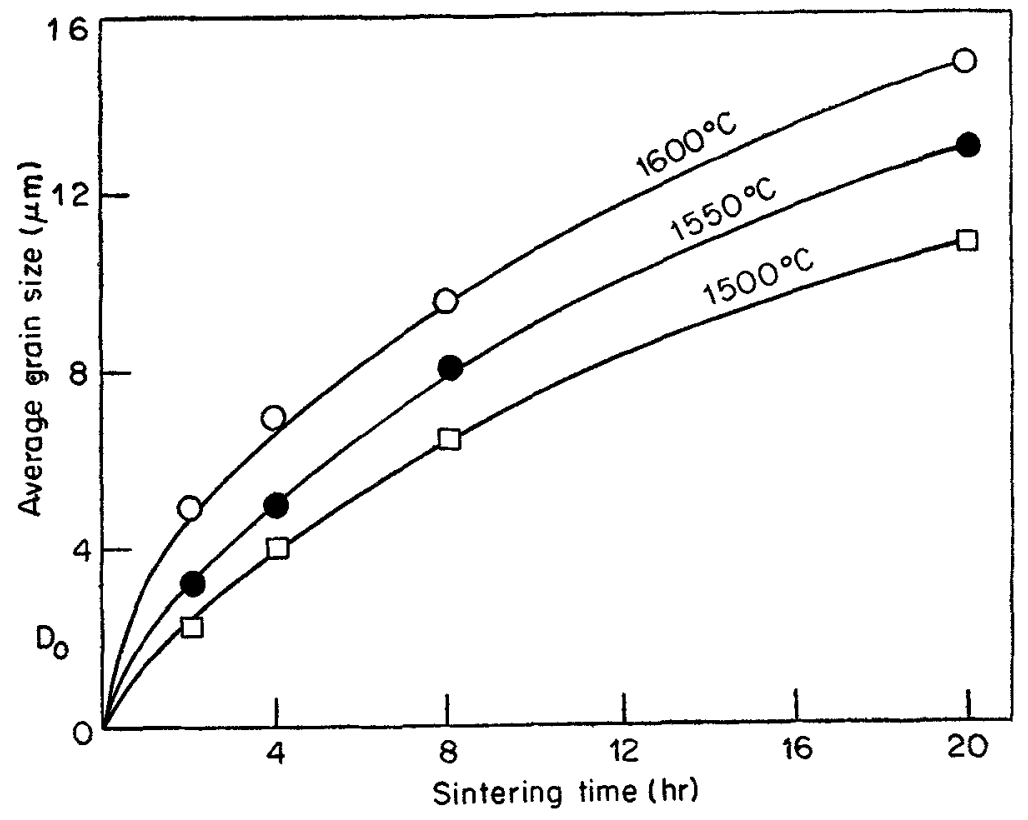

Figure 5. Grain growth isotherm for LCT-3 composition.

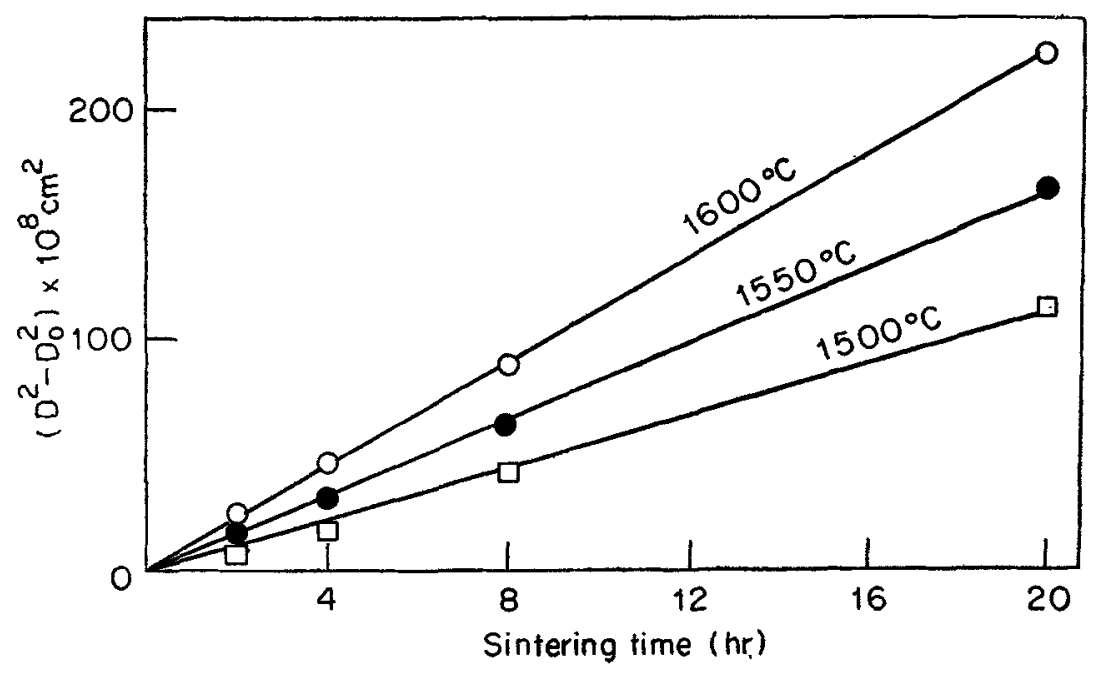

Figure 6. Plots of the equation $D^{2}-D_{0}^{2}=k t$ for LCT-3.

in higher grain size. Hence the addition of $\mathrm{TiO}_{2}$ leads to higher densification and increased grain growth rate in $\mathrm{LaCrO}_{3}$.

\section{Conclusions}

(i) $3 \mathrm{wt} \% \mathrm{TiO}_{2}$ additions improves the sintering of $\mathrm{LaCrO}_{3}$ and densities $>90 \%$ of the theoretical value are achieved. 


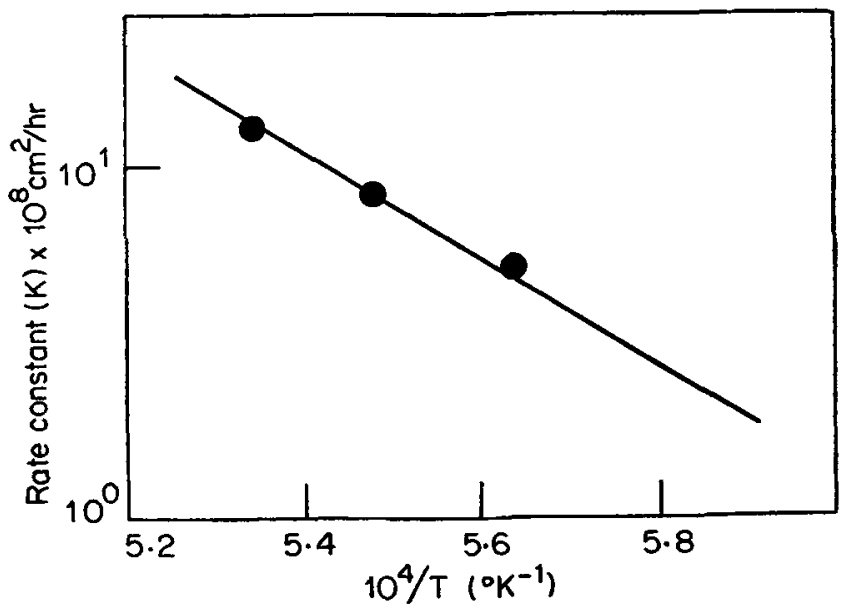

Figure 7. Arrhenius plot of rate constant $K$ versus (1/T) for LCT-3 composition.

(ii) Enhanced densification of $\mathrm{LaCrO}_{3}$ with the addition of titania is best explained as due to the creation of cation vacancies.

\section{Acknowledgements}

One of the authors (KPB) wishes to acknowledge UGC for extending financial assistance in carrying out the present work. The assistance of Mrs U Dhawan in taking $\mathrm{x}$-ray diffractograms is gratefully acknowledged.

\section{References}

ASTM Designation 1971, C20-70 Parts 136.

Anderson $\mathrm{H}$ U et al 1977, 13th Rare Earth Conf, Olgebay Park W. Vir, Oct. 16

Anderson H U 1978 Processing of crystalline ceramics (New York: Plenum) 467-77

Anderson H U 1974 J. Am. Ceram. Soc. 5734

Bansal K P, Kumari S, Das B K and Jain G C 1980 Communicated to Tran. J. Brit. Ceram. Soc. Callister W D et al $1979 \mathrm{~J}$. Am. Ceram. Soc. 62208

George A M and Karkhanawala M D 1979 Symp. Sintering \& Sintered Products BARC, Oct. 29-31

Halloran J W and Anderson H U $1974 \mathrm{~J}$. Am. Ceram. Soc. 57150

Khattak C P and Cox D E 1977 Mat. Res. Bull. 12463

Leslie Group and Anderson H U 1976 J. Am. Ceram. Soc. 59449

Meadowcroft D B 1969 Br. J. Appl. Phys. Ser. 21225

Meadowcroft D B 1972 Energy Conversion 8185

Meadowcroft D B 1972 Energy Conversion 12145

Meadowcroft D B 1973 Conf. on Strontium Containing Compounds, Halifex, Canada

St. Jacques R G, Moise A and Yerouchalmi D 1974 J. Canad. Ceram. Soc. 4323

Stuijts A L 1968 Ceramic Microstructures Chap. 19 (New York: John Wiley)

Yan M F and Johnson Jr D W 1978 J. Am. Ceram. Soc. 61342 